\title{
Theory and applications of swarm intelligence
}

\author{
Zhihua Cui $\cdot$ Xiaozhi Gao
}

Published online: 29 January 2011

(C) Springer-Verlag London Limited 2011

Swarm intelligence is the discipline that deals with natural and artificial systems composed of many individuals that coordinate their activities using decentralized control and self-organization. In particular, the discipline focuses on the behavior of social insects such as fish schools and bird flocks and colonies of ants, termites, bees, and wasps. The most well-known examples of systems studied by swarm intelligence are particle swarm optimization (PSO) and ant colony optimization (ACO).

Particle swarm optimization mimics the behavior of fish schooling and bird flocking. PSO is a population-based stochastic optimization strategy with fast convergent speed than general evolutionary algorithms (EAs). Different from EAs, each particle employs not only position information but also the velocity information. They communicate good positions to each other and adjust their own positions according to their decision. In PSO, a number of simple entities - the particles - are placed in the search space of some problem or function, and each evaluates the objective function at its current location. Each particle then determines its movement through the search space by combining some aspect of the history of its own current and best (best-fitness) locations with those of one or more members

\section{Z. Cui ( $\square)$}

Complex System and Computational Intelligence Laboratory,

Taiyuan University of Science and Technology,

030024 Shanxi, Taiyuan, China

e-mail: cuizhihua@gmail.com

\section{Z. Cui}

State Key Laboratory for Novel Software Technology,

Nanjing University, 210093 Nanjing, Jiangsu, China

X. Gao

Aalto University, Aalto, Finland

e-mail: gao@cc.hut.fi of the swarm, with some random perturbations. The next iteration takes place after all particles have been moved. Eventually, the swarm as a whole, like a flock of birds collectively foraging for food, moves close to an optimum of the fitness function.

Ant colony optimization is a probabilistic technique for solving computational hard problems which can be reduced to finding optimal paths. The main idea is the indirect communication between the ants by means of chemical pheromone trails.

The special issue on the swarm intelligence is dedicated to the latest work in the theory and applications in this exciting area. Our aim is to provide a useful reference for understanding new trends on swarm intelligence. After a detailed review process, a total of four papers were selected to reflect the call thematic vision. The contents of these studies are briefly described as follows.

The first paper entitled 'Analysis of emergent symmetry breaking in collective decision making' by Heiko Hamann, Thomas Schmickl, Heinz Worn, and Karl Crailsheim investigates a simulated multi-agent system (MAS) that collectively decides to aggregate at an area of high utility. The agents' control algorithm is based on random agentagent encounters and is inspired by the aggregation behavior of honey bees. In this article, symmetry breaking is defined, several symmetry-breaking measures are proposed, and phenomenon of emergent symmetry breaking within authors' observed system is reported. The ability of the MAS to successfully break the symmetry depends significantly on a local-neighborhood-based threshold of the agents' control algorithm that determines at which number of neighbors the agents stop. This dependency is analyzed, and two macroscopic features are determined that significantly influence the symmetry-breaking behavior. In addition, the connection between the ability of the 
MAS to break symmetries and the ability to stay flexible in a dynamic environment is also investigated.

In recent years, a few sequential covering algorithms for classification rule discovery based on the ant colony optimization meta-heuristic (ACO) have been proposed. In the paper, 'A correlation based ant miner for classification rule discovery', Abdul Rauf Baig and Waseem Shahzad propose a new ACO-based classification algorithm called AntMiner-C. Its main feature is a heuristic function based on the correlation among the attributes. Other highlights include the manner in which class labels are assigned to the rules prior to their discovery, a strategy for dynamically stopping the addition of terms in a rule's antecedent part and a strategy for pruning redundant rules from the rule set. The performance of proposed approach for twelve commonly used datasets is investigated and compared it with the original Ant-Miner algorithm and a decision tree builder, C4.5. Experimental results show that the accuracy rate obtained by Ant-Miner-C is better than that of the compared algorithms and is also better compared to the results reported in literature for other ACO-based classification algorithms.

Particle swarm optimization (PSO) has recently emerged as a nature-inspired algorithm for real parameter optimization. In the paper, 'An inertia-adaptive particle swarm system with particle mobility factor for improved global optimization', Sayan Ghosh, Swagatam Das, Debarati Kundu, Kaushik Suresh, B. K. Panigrahi, and Zhihua Cui describe a method for improving the final accuracy and the convergence speed of PSO by first adding a new coefficient (called mobility factor) to the position updating equation and second modulating the inertia weight according to the distance between a particle and the globally best position found so far. The twofold modification tries to balance between the explorative and exploitative tendencies in PSO to achieve better search performance. The effect of the modifications on the dynamics of the PSO algorithm is also mathematically analyzed. The new algorithm has been shown to be statistically significantly better than four state-of-the-art variants of PSO on a ten-function test-bed and a real life engineering optimization problem.

For honey bee colonies, it is crucial to collect nectar in an efficient way. Empiric experiments showed that the process of decision making, which allows the colony to select the optimal nectar source, is based purely on individual decisions. These decisions are made by returning nectar foragers, which alter their dancing behaviors based on the nectar source's quality and based on the experienced search time for a receiver bee. Nectar receivers, which represent a shared limited resource for foragers, can modulate the foraging decisions performed by the colony. In the paper,

'Swarm-intelligent foraging in honey bees: benefits and costs of task-partitioning and environmental fluctuations', Thomas Schmickl, Ronald Thenius, and Karl Crailsheim investigate the interplay between foragers and receivers by using a multi-agent simulation, agents which are capable of a limited set of behaviors and which spend energy according to their behavior are implemented. In simulation experiments, colonies with various receiver-to-forager ratios are tested and colony-level results like the emerging for aging patterns and the colony's net honey gain are measured. Results show that the number of receivers prominently regulates the foraging work force. All environmental fluctuations are predicted to cause energetic costs for the colony. Task-partitioning additionally influences the colony's decision making whether to stick to a nectar source after such environmental fluctuations.

The guest editors of this special issue in Neural Computing \& Applications would like to thank all authors for submitting their interesting work. We are grateful to the reviewers for their great contributions to this special issue. We would like to express our sincere appreciation to the Editors-in-Chief, Prof. John MacIntyre, for his advice and support. This special issue has been supported by National Natural Science Foundation of China under Grant 61003053, the Key Project of Chinese Ministry of Education under Grant 209021 and Shanxi Science Foundation for Young Scientists under Grant 2009021017-2. 